\title{
Loss of Tissue Regenerative Capacity in Aging - The Tendon
}

\section{Pedro Carvalho Sá ${ }^{1 *}$, Margarida Pimenta Queiroz Valério ${ }^{2}$, Inês Monteiro Ferro ${ }^{3}$ Inês Campos $^{4}$ and Lina Carvalho ${ }^{5}$}

${ }^{1}$ Physical Medicine and Rehabilitation Resident, $4^{\text {th }}$ year; Centro de Medicina de Reabilitação da Região Centro - Rovisco Pais, Tocha, Portugal

${ }^{2}$ Pulmonology Resident, $5^{\text {th }}$ year; Serviço de Pneumologia do Centro Hospitalar e Universitário de Coimbra, Coimbra, Portugal

${ }^{3}$ Physical Medicine and Rehabilitation Resident, $4^{\text {th }}$ year; Centro de Medicina de Reabilitação da Região Centro - Rovisco Pais, Tocha, Portugal

${ }^{4}$ Physical Medicine and Rehabilitation Specialist; Centro de Medicina de Reabilitação da Região Centro Rovisco Pais, Tocha, Portugal

${ }^{5}$ Pathology Specialist; Serviço de Anatomia Patológica do Centro Hospitalar e Universitário de Coimbra, Coimbra, Portugal

*Corresponding author: Pedro Carvalho Sá, Medical Resident of Physical and Rehabilitation Medicine in Portugal, Currently in the $4^{\text {th }}$ Year of Residency at Centro de Medicina de Reabilitação da Região Centro, Rovisco Pais, Portugal

\begin{abstract}
Intrinsic to the process of aging is the loss of the ability to regenerate of different organs and tissues, becoming more susceptible to aggression and impaired function. Tendon aging is a complicated process following, however, similar mechanisms of other tissues - proposed model of the "Hallmarks of Aging", by López-Otín, et al [1]. Tendon structure and cellular composition is also unique, which makes scientific research in this field both a very specific and select task. Mesenchymal Stem Cells (MSCs), more specifically, Tendon stem/progenitor cells (TSPCs) may be of key importance.

Tendon disease is one of the most common entities worldwide, and is currently increasing with the augment of the human life-expectancy. This review proposes to make a short summary of the state of the art in both the aging tendon and its available treatment target potentials.
\end{abstract}

\section{Introduction}

Aging is characterized by a progressive loss of physiological integrity, leading to impaired function and increased vulnerability to death [1]. This inherited life characteristic heterogenous form organs to tissues, where tendons as highly organized connective tissues comprise bottom-up assemblies of collagen molecules [2].
Tendon cells include tenocytes are differentiated cells committed with synthesis of scleraxis (Scx), a basic loop-helix-loop transcription factor; tenomodulin $+($ Tnmd), a transmembrane glycoprotein, as well as with the tendon extracellular matrix (ECM) component collagen type I (Col-1) together with collagen type III, proteoglycans and glycosaminoglycans. Tendon stem/ progenitor cells (TSPCS) fulfill the universal criteria of mesenchymal stem cells (MSCs): Clonogenicity, multipotency and self-renewal according with stimuli and respective cellular adaptation.

Tendon aging is a complicated process caused by multifaceted pathways and aging plays a critical role in the occurrence and severity of tendon injury [3].

Due to an increasingly aged population, the occurrence of tendon-related injuries is also increasing. No significant advances have been made in the treatment of such conditions in recent years.

The purpose of this review is to is discuss the current evidence on tendon aging, its treatment, and how this can eventually create new targets for therapy.

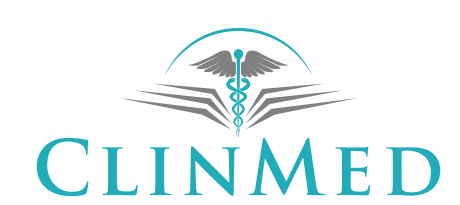

INTERNATIONAL LIBRARY
Citation: Sá PC, Valério MPQ, Ferro IM, Campos I, Carvalho L (2020) Loss of Tissue Regenerative Capacity in Aging - The Tendon. Int J Stem Cell Res Ther 7:071. doi.org/10.23937/2469-570X/1410071 Accepted: December 08, 2020; Published: December 10, 2020

Copyright: (c) 2020 Sá PC. This is an open-access article distributed under the terms of the Creative Commons Attribution License, which permits unrestricted use, distribution, and reproduction in any medium, provided the original author and source are credited. 


\section{Material and Methods}

An analytical review conducted through PubMed database relied on original articles using "tendon cell aging" and "tendon cell regeneration" as main objectives. The search included only English written articles, without year of publication restrictions. The articles selected included those that reflected studies/reviews of aging mechanisms in a specific tissue: The tendon, evolving historical summing up knowledge, conductivity to cellular and matrix crosslinking, in order to allow medical understanding of the disease and its potential therapeutic targets.

\section{Loss of Tissue Regenerative Capacity in Aging}

Models to explain the process of aging have been proposed throughout years. In 2013 Carlos Lopéz-Otin, et al. proposed "The Hallmarks of Aging" enumerating nine hallmarks that represent common denominators of aging: Genomic instability, telomere attrition, epigenetic alterations, loss of proteostasis, deregulated nutrient-sensing, mitochondrial dysfunction, cellular senescence, stem cell exhaustion, and altered intercellular communication [1].

Deficient proliferation of stem/progenitor cells seemed to be detrimental the long-term resistance, while excessive proliferation might be deleterious by accelerating the exhaustion of the stem cell niches [1].

TSPCs represent a minor percentage of the overall tendon cell composition. These cells present with features such as self-renewal, clonogenicity and multidifferentiation to be distinguished through the presence of stem cell markers [3].

Up-regulation of P53, P16, P14 and P21 genes related to cell cycle arrest and activation of the P53 and P21 pathways have been observed in aged TSPCs, which are thought to function as accelerators of the cellular ageing process [4]. The inbalance in between suppressor genes seems to conduct to cell exhaustion in the tendon tissue.

Epigenetic changes involving alterations in DNA methylation patterns, post-translational modification of histones, and chromatin remodelling [1] support a series of significant cellular epigenetic alterations through age, viewed as age-related markers in TSPCs. These results are consistent with those obtained from other types of stem cells [3] and can be used in future studies.

\section{Tendon Stem/Progenitor Cells}

Pluripotent stem cells are supposed to differentiate into various tissue types under different genetic stimuli/ conditions and serve as an internal repair system, according with embryonic layer of origin [3].

Compared with bone marrow stromal cells, TSPCs express higher levels of Oct 4, known to positively modulate mesodermal lineage differentiation and have greater ability to proliferate and get clonogenicity. Thus, TSPCs potentially represent a more appropriate cell source for the regeneration of musculoskeletal tissue, particularly tendon tissue, which has limited repair and healing abilities with traditional tenocytes [3].

Aging and Alterations in Epigenetic and Underlying Mechanisms

\section{Aging and cell morphology}

In vitro aged TSPCS exhibit cell star-like flattened shape, while youngTSPCs exhibit spindle-shaped morphology [4]. In addition, aged TSPCS arelarger in size, have more podia, spread further, and exhibit more robustactin stress fibers and a higher actin content that distorts the balance of the actincytoskeleton organization [4]. An increase in the size is often associated with cell senescence [5]. Additionally, aged TSPCs display a large, flat andheterogeneous morphology, while younger cells exhibit an uniform elongated morphology [6].

\section{Aging and cell differentiation}

The capacity of TSPCs to differentiate into tenocytes is reduced with ageing [7] consistent with the observation that capacity of differentiation of the TSPCs is markedly diminished duringthe ageing process. Moreover, aged TSPCs are not sensitive to transforming growth factor- $\beta 3$, a sublineage of the TGF- $\beta$ superfamily that regulates cell growth and differentiation also engaged in matrix renewal [8].

Although,TSPCs have been reported to display a decrease in self-renewal and clonogenic capacities, multipotency has been maintained in vitro [4].

Multidifferentiation capacities of TSPCS are maintained during ageing process without a conclusive determination of the trends in their variations, but most studies conclude that aging impairs the tenogenic differentiation capacity of TSPCs [3].

\section{Aging and cell proliferation}

According to Docheva, et al. (2005) [9], Tnmd is an established gene marker for the mature tendon/ligament lineage in vertebrates. Its protein has a highly conserved cleavable C-terminal cysteine-rich domain that has been identified as an important regulator for TSPCS proliferation and senescence as well as for tendon maturation. Tnmd loss-of-function in mice leads to a phenotype with distinct signs of premature aging on tissue and stem/progenitor cell levels [10].

Aged TSPCs showed a proliferation deficit after 120 days of culture and had an early plateau phase, while young TSPCs did not exhibit the plateau [4]. It has alsobeen observed this decrease in TSPCs proliferation with increasing age, consistent withsimilar results observed in TSPCs from other aged vertebrate animals [5].

Another age-dependent important factor, is the ca- 
pacity for these cells to form colonies (clonogenicity). Globally, clonogenic deficits in TSPCs are based in reduced colonynumber and lowering colony-forming unit efficiency verified in aging.

\section{Aging and cell viscoelasticity}

A variety of tissues express Tnmd mRNA, however Tnmd cleavage and secretion is exclusive to tissues undergoing tension, suggestive of a possible mechano-regulation mechanism [10].

Stiffening of human tendons was observed in aging or in diabetic individuals due to increased pathological cross-linking of collagen fibrils [11].

Denser, well-structured actin cytoskeleton in aged TSPC, seems to correlate with augmented cell stiffness [12]. Also a dense cytoskeletal organization might result in a larger cell size and anomalous cell shape conducting also to increase stiffness and viscosity [6].

\section{Mechanisms involved in the aging process}

Despite unclear molecular mechanisms involved, aging is known as a risk factor for tendon disorders. When TSPCs premature enter into senescence exhibit proliferation, differentiation and differentiation deficits. Many pathways and theories have been proposed up to date [3].

Forkhead box P1 (FOXP1) gene belongs to subfamily $P$ of the fork head box (FOX) transcription factor family and plays important roles in various biological processes, including cell cycle progression, proliferation, and differentiation and senescence [13]. During TSPCs aging, FOXP1was significantly reduced in both MRNA and protein levels; age-related dysfunction, such as self-renewal, migration and differentiation, appear to be a hallmark of TSPCs senescence [13].

There are also different mechanisms of the aged tendon to adapt to stressors with aging. The effect of these biological changes on the ability of resident tenocytes to respond to various stimuli is still relatively unexplored and present many avenues for future research suggesting differential pathways in males and females, supporting matrix turnover in males (increased matrix degeneration, apoptosis) but tissue preservation in females (decreased inflammation, apoptosis, matrix degeneration) [14].

\section{Rejuvenation of aged tendon (Stem/Progenitor Cells)}

Numerous factors are involved in altering tendon homeostasis. Macroscopic factors associated with an uncomfortable exercise intensity and microscopic factors associated with an impaired estrogen balance, deteriorated ECM conditions and inappropriate drug use, alter the features of TSPCs, particularly during aging [3].

Based on most recent development in regenerative medicine, Dale, et al. [15] induced human embryonic stem cells to differentiate into tendon-like cells in the presence of exogenous bone morphogenetic protein (BMP) 12 and BMP 13 and directed parthenogenetic stem cells to differentiate into tenocytes, similar to embryonical development.

\section{Discussion and Conclusions}

In human musculoskeletal injuries, specifically in tendon injuries, there is no optimal treatment strategy till this day. TSPCs can be the beginning of a new era in the treatment of these kind of injuries - either by being stimulated or by being transplanted.

Evidence suggests that tendon resident cells, including tendon MSCs (including TSPCS) may be the main orchestrators directing tendon-regenerative processes. Such biological response upon injury may be further boosted by the administration of non-tendon MSCs.

An important role for increased Rho has been reported, associating coiled-coil forming protein kinase (ROCK) activity in accelerating the ageing progress of aged TSPC, changing back to a morphology similar to young TSPCs upon treatment with Y-27632, a common ROCK inhibitor [4]. These changes which are observed in tendon with increasing age, may inherit a high density of intrafibrillar covalent cross-links and this could regulate tensile fracture resistance mechanics [16], making it more rigid and less elastic - propitious to rupture. Treating aged TSPC with ROCK-inhibitor, might reverse these age-related changes and rejuvenating effect on cell morphology and stiffness may be acquired. We assume that cellular stiffness is a suitable marker for cell aging and ROCK a potential target for therapeutic applications of cell rejuvenation [12] - also a target for further research in the future.

As important as it is to understand the mechanisms of aging in the tendon, it is also paramount to understand how does the aged tendon respond to different stressors.

Moderate exercise ameliorates the depletion of the TSPC pool by upregulating the expression of cell proliferation and stem cell markers coupled with decreased lipid deposition, proteoglycan accumulation and calcification formation, and it is beneficial for delaying the undesirable effects of age [17].

Recently, the discovery of induced pluripotent stem cells (iPSCs), particularly cells isolated from mature adult, inspired researchers to develop potential therapies to cure clinical diseases and ponder the eternal topic of regaining our youth. Thus, iPSCs provided inspiration to reverse the stem cell fate by modulating the factors that influence cell growth [3]. Mechanical stretching improved the tenogenic differentiation of both these iPSCs and pre-MSCs [18].

When tenocytes are exposed to a certain amount 
NSAIDs, the differentiation of MSCs to tenocytic lineage gets impaired and drawn toward adipocytic lineage [19]. Different concentrations of ascorbic acid have been used in tenocyte cultures to enhance collagen synthesis - ascorbic acid is a well-characterized antioxidant that can promote collagen biosynthesis and prevent free radical formation [19]. Because of its acidity, the exact concentration of ascorbic acid to use is still unknown.

Inflammation does not seem to affect the proliferation rate of the isolated TSPCs and the tenogenic marker gene expression [20]. Supporting the theory: that the long head of the biceps tendon with and without tendinitis may be a novel source of tendon derived stem cells, which might facilitate treatment of degeneration and induction of regeneration in shoulder surgery, like J Schmalzl, et al. [20] proposed.

Pioglitazone can be used to enhance the therapeutic effects of mesenchimal stem cells for tendon repair, as shown by the increased proliferation, migration, remolding gene expression, and ECM secretion of tenocytes [21].

Various types of biophysical stimulations, such as ultrasonication and shock wave, were experimentally confirmed to be beneficial for promoting the differentiation and proliferation of MSCs [22].

As an effective, non-invasive, cheap, and safe ultrasonication, low-intensity pulsed ultrasound (LIPUS) was shown to accelerate bone-tendon healing, the mechanism of which might be related to its influence on the local mechanical and biologic environment and thus its ability to induce lineage-specific differentiation of the MSCs around the healing interface [22].

To this date, tendon aging can be correlated with TSPC aging. Limited studies, have been done to explore all theelaborate cell repair mechanisms in the tendon.

As life expectancy augments, so do tendon injuries. No inovative treatments/strategies have appeared in recent years. It is of atmost importance that we understand effectively how does in fact the tendon ages and adjust accordingly: Alternative guided treatment strategiesthat can be offered to clinicians around the globe.

\section{References}

1. López-Otín C, Blasco MA, Partridge L, Serrano M, Kroemer $G$ (2013) The hallmarks of aging. Cell 153: 1194-1217.

2. Costa-Almeida R, Calejo I, Gomes M (2019) Mesenchymal stem cells empowering tendon regenerative therapies. Int J Mol Sci 20: 3002.

3. Dai GC, Li YJ, Chen MH, Lu PP, Rui YF (2019) Tendon stem/progenitor cell ageing: Modulation and rejuvenation. World J Stem Cells 11: 677-692.

4. Kohler J, Popov C, Klotz B, Alberton P, Prall WC, et al. (2013) Uncovering the cellular and molecular changes in tendon stem/progenitor cells attributed to tendon aging and degeneration. Aging Cell 12: 988-999.

5. Popov C, Kohler J, Docheva D (2016) Activation of EphA4 and EphB2 reverse signaling restores the age-associated reduction of self-renewal, migration, and actin turnover in human tendon stem/progenitor cells. Front Aging Neurosci 7: 246 .

6. Wu H, Zhao G, Zu H, Wang JH, Wang QM (2015) Aging-related viscoelasticity variation of tendon stem cells (TSCs) characterized by quartz thickness shear mode (TSM) resonators. Sens Actuators (Warrendale Pa) 210: 369-380.

7. Chen J, Zhang W, Liu Z, Zhu T, Shen W, et al. (2016) Characterization and comparison of post-natal rat Achilles tendon-derived stem cells at different development stages. Sci Rep 6: 22946.

8. Kovacevic D, Rodeo SA (2008) Biological augmentation of rotator cuff tendon repair. Clin Orthop Relat Res 466: 622633.

9. Docheva D, Hunziker EB, Fassler R, Brandau O (2005) Tenomodulin is necessary for tenocyte proliferation and tendon maturation. Mol Cell Biol 25: 699-705.

10. Dex S, Alberton P, Willkomm L, Söllradl T, Bago S, et al. (2017) Tenomodulin is required for tendon endurance running and collagen I fibril adaptation to mechanical load. E BioMedicine 20: 240-254.

11. Couppe C, Svensson RB, Kongsgaard M, Kovanen V, Grosset JF, et al. (2016) Human Achilles tendon glycation and function in diabetes. J Appl Physiol 120: 130-137.

12. Kiderlen S, Polzer C, Rädler JO, Docheva D, Clausen-Schaumann $\mathrm{H}$, et al. (2019) Age related changes in cell stiffness of tendon stem/progenitor cells and a rejuvenating effect of ROCK-inhibition. Biochem Biophys Res Commun 509: 839-844.

13. Xu H, Fan Liu F (2018) Downregulation of FOXP1 correlates with tendon stem/progenitor cells aging. Biochem Biophys Res Commun 504: 96-102.

14. Connizzo BK, Piet J, Sandra J Shefelbine, Alan J Grodzinsky (2019) Age-associated changes in the response of tendon explants to stress deprivation is sex-dependent. Connect Tissue Res 61: 48-62.

15. Dale TP, Mazher S, Webb WR, Zhou J, Maffulli N, et al. (2018) Tenogenic differentiation of human embryonic stem cells. Tissue Eng Part A 24: 361-368.

16. Goh KL, Holmes DF, Lu YH, Kadler KE, Purslow PP (2018) Age-related dataset on the mechanical properties and collagen fibril structure of tendons from a murine model. Sci Data 5: 180140.

17. Zhang J, Wang JH (2015) Moderate exercise mitigates the detrimental effects of aging on tendon stem cells. PLoS One 10: e0130454.

18. Liu W, Yin L, Yan X, Cui J, Liu W, et al. (2017) Directing the differentiation of parthenogenetic stem cells into tenocytes for tissue-engineered tendon regeneration. Stem Cells Transl Med 6: 196-208.

19. Chiu CH, Chen P, Chen AC, Chan YS, Hsu KY, et al. (2019) Real-time monitoring of ascorbic acid-mediated reduction of cytotoxic effects of analgesics and NSAIDs on tenocytes proliferation. Dose-Response 17: 1559325819832143.

20. J SchmalzI, P Plumhoff, F Gilbert, F Gohlke, C Konrads, et al. (2019) Tendon-derived stem cells from the long head of the biceps tendon. Bone Joint Res 8: 414-424.

21. Kim W, Lee SK, Kwon YW, Chung SG, Kim S (2019) Pioglitazone-primed mesenchymal stem cells stimulate cell proliferation, collagen synthesis and matrix gene expression in tenocytes. Int J Mol Sci 20: 472.

22. Can Chen, Tao Zhang, Fei Liu, Jin Qu, Yang Chen, et al. (2019) Effect of low-intensity pulsed ultrasound after autologous adipose-derived stromal cell transplantation for bone-tendon healing in a rabbit model. Am J Sports Med 47: 942-953. 